\title{
VAN DER WAals EquATION OF STATE
}

PROBLEM: Infinite susceptibility coefficients $(\alpha, \kappa)$ obstruct the simulation of phase transitions under natural (isobar/isotherm) conditions, until now. SOLUTION: The combination of the Time Dependency with the Van der Waals Equation of State (as a blue print for other EoS) allows corresponding simulations of CROSSING PHASE TRANSITION POINTS by the new DIFFERENTIAL SIMULATED ANNEALING/COMPRESSING methods.

Van der Waals Equation of State

(J.D. van der Waals, Ph.D. thesis (1873))

$$
\begin{aligned}
& \left(p_{0}+\frac{a}{V_{m, 0}^{2}}\right)\left(V_{m, 0}-b\right)=N_{A} k_{B} T_{0} \\
& a=\frac{27}{64} \frac{R^{2} T_{c r}^{2}}{p_{c r}} \quad b=\frac{1}{8} \frac{R T_{c r}}{p_{c r}} \\
& p_{r}=\frac{p_{0}}{p_{c r}} \quad V_{m, r}=\frac{V_{m, 0}}{V_{m, c r}} \quad T_{r}=\frac{T_{0}}{T_{c r}} \\
& \left(p_{r}+\frac{3}{V_{m, r}^{2}}\right)\left(3 \cdot V_{m, r}-1\right)=8 \cdot T_{r}
\end{aligned}
$$

\section{Time dependency}

(H. Berendsen, J.Chem.Phys. (1984))

\section{Total Time Differentials}

(Brondstein, Verlag Harri Deutsch, (2013))

$$
\begin{gathered}
\lambda=\left[1+\frac{\Delta t}{\tau_{T}}\left\{\frac{T_{0}}{T(t-0.5 \Delta t)}-1\right\}\right]^{\frac{1}{2}} \\
\mathrm{p}(t)=\frac{2}{3 V}\left(\mathrm{E}_{k i n}(t)-\Xi(t)\right) \\
\mu(t)=1-\frac{\Delta t}{\tau}\left(p_{0}-p(t)\right)
\end{gathered}
$$

$V(t+\Delta t)=\mu V(t)$

(5) $\frac{\delta F}{\delta p_{r}} \frac{d p_{r}(t)}{d t}+\frac{\delta F}{\delta V_{m, r}} \frac{d V_{m, r}(t)}{d t}+\frac{\delta F}{\delta T_{r}} \frac{d T_{r}(t)}{d t}=0$

(6) $0=\left(3 V_{m, r}^{3}-V_{m, r}^{2}\right) \frac{d p_{r}(t)}{d t}$

$$
\begin{aligned}
& +\left(9 p_{r} V_{m, r}^{2}-2\left(p_{r}+8 T_{r}\right) V_{m, r}+9\right) \frac{d V_{m, r}(t)}{d t} \\
& +\left(-8 V_{m, r}^{2}\right) \frac{d T_{r}(t)}{d t}
\end{aligned}
$$

Rearrangement gives explicit fracture expres(8) sions for $\frac{d p_{r}(t)}{d t}, \frac{d V_{m, r}(t)}{d t}$ and $\frac{d T_{r}(t)}{d t}$

\section{Some additional mathematical considerations}

\section{Some common definitions}

$$
\begin{gathered}
\frac{d x}{d t}=-\left(\frac{\frac{\delta F}{\delta y}}{\frac{\delta F}{\delta x}}\right)_{z=c o n s t} \frac{d y}{d t} \\
\frac{d x(t)}{d y(t)}=\frac{1}{\frac{d y(t)}{d x(t)}}
\end{gathered}
$$

$$
\begin{aligned}
\phi(t)= & \frac{1}{x(y(t))} \frac{d x(t)}{d y(t)}=\frac{d \ln x(y(t))}{d y(t)}=-\frac{1}{x(y(t))}\left(\frac{\frac{\delta F}{\delta y}}{\frac{\delta F}{\delta x}}\right)_{z=c o n} \\
& x_{i+1} \approx x_{i}-\left(\frac{\frac{\delta F}{\delta y}}{\frac{\delta F}{\delta x}}\right)_{z=\text { const },_{i}}\left(\frac{d y}{d t}=\text { const. }=k_{d y}\right) \Delta t
\end{aligned}
$$

Functions and their inverse functions

$$
x(y, z=\text { const }) \quad \text { and } \quad y(x, z=\text { const })
$$

1. monotonic increasing: $\quad p_{r}\left(T_{r}, V_{m, r}=\right.$ const $)$ and $T_{r}\left(p_{r}, V_{m, r}=\right.$ const $)$;

2. unambigous with extrema: $T_{r}\left(V_{m, r}, p_{r}=\right.$ const $)$ and $p_{r}\left(V_{m, r}, T_{r}=\right.$ const $)$

3. ambigous: $\quad V_{m, r}\left(T_{r}, p_{r}=\right.$ const $)$ and $V_{m, r}\left(p_{r}, T_{r}=\right.$ const $)$

Important (Bronstein at page 52): Cite: "If there is a function with non-monotonic behavior separable into monotonic pieces then the corresponding inverse function exists for every of that monotonic piece."

$$
f(x)=y \quad<==>\quad f(y)=x
$$

Three special cases Isochor $-\frac{d V_{m, r}}{d t}=0$
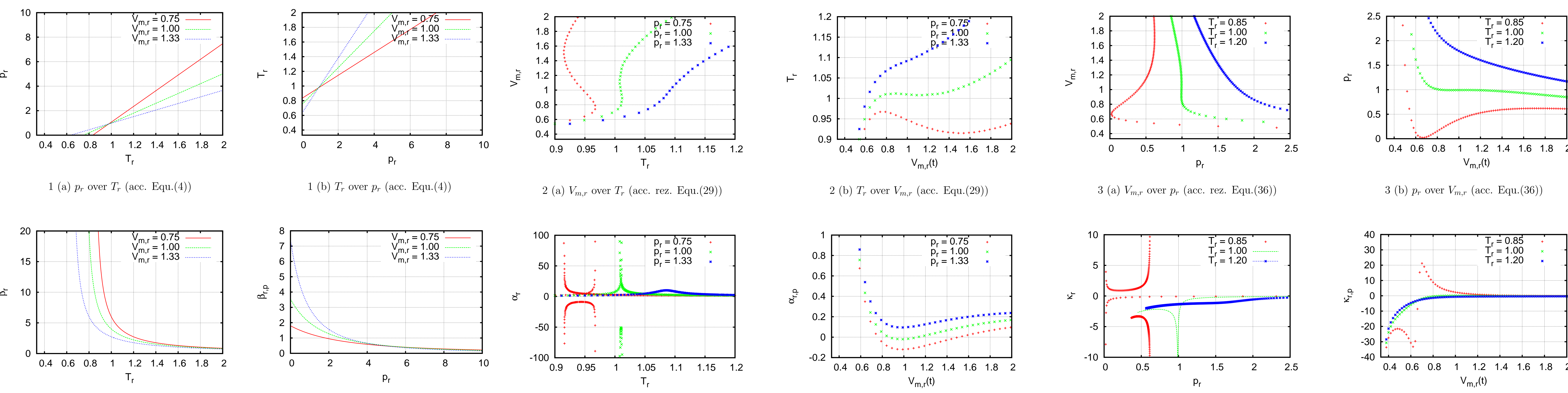